\title{
A Dosimetric study of different MLC expansion aperture For the radiotherapy of pancreas cancer
}

\author{
Mohammad F. Hassan*, Mahmoud A. Hefni **, Mohamed El-Azab Farid **, \\ Khaled M. Elshahat *** \\ * Assuit Military center for Radiotherapy, Assuit, Egypt. \\ ** Physics Department - Faculty Sciences - Assuit University, Assuit, Egypt. \\ *** Radiation Oncology department-Faculty of Medicine - Al Azhar University, Cairo, Egypt.
}

\begin{abstract}
Radiotherapy is using of ionizing radiation in the treatment of cancer. The aim of radiotherapy is to annihilate the tumor tissue while minimizing damage to the normal surrounding tissues. We can do that by using shielding like custom blocks and MLCs. Uses the MLCs now in all the sites, but does not use blocks except in the necessity of it, as there are different types of MLCs in thickness $(1 \mathrm{~cm}$ and $0.5 \mathrm{~cm}$, and finally $0.3 \mathrm{~cm})$. The MLCs are not limited to the use of three-dimensional planning only, but also using in the most modern techniques like IMRT and IGRT. When shaping the radiotherapy treatment field size of the tumor, there are some concepts must be included in the accounts, such as the concept of penumbra and radiotherapy field size and the impact of change that field size on the dose distribution within the tumor and dose receiving by OARs. When the field size is shaped where the MLCs ends are attached with tumor (the MLCs margin $=0.0 \mathrm{~cm}$ ) the penumbra effects on the tumor cover percentage, so the distance between tumor and MLCs ends (MLC margins) must be existing to overcome the penumbra effects. To determine the most adequate MLC margins which achieve the tumor dose coverage a many plans with difference MLC margins were compared from $0.0 \mathrm{~cm}$ to 1.2 $\mathrm{cm}$ the comparison included the tumor coverage percentage and the received dose to OARs and if it under tolerance or not. After that we can selection the best MLC margins to work out in most famous techniques for pancreas cases.
\end{abstract}

Results: the tumor is not cover when the MLC margins less than $0.5 \mathrm{~cm}$, and from MLC margins $0.5 \mathrm{~cm}$ to 0.7 $\mathrm{cm}$ the tumor is cover very well and OARs are under tolerance, the MLC margins more than $0.7 \mathrm{~cm}$ the tumor cover is very well but OARs received unnecessary doses. After $1.2 \mathrm{~cm}$ MLC margin, the saturation occurs of the tumor cover percentage.

Conclusions: The best MLC expansion apertures for the radiotherapy of pancreas cancer are from $0.5 \mathrm{~cm}$ to $0.7 \mathrm{~cm}$.

\section{Introduction}

Radiotherapy is one of the three principal modalities used in the treatment of malignant disease (cancer), the aim of radiotherapy is to kill cancer cells by deliver as much dose as possible to the target while minimizing the dose to surrounding healthy tissues by radiotherapy machines like linear accelerator which exits the radiation as certain area called field size determined by collimator jaws. These fields must take the shape of the tumor as much as possible; there are many ways to do that ether using custom blocks or using MLCs.

The using of MLCs has many advantages and become the most common in modern devises. When the MLCs is used to shape field size around tumor we must take the penumbra in our accounts and then we should chose adequate MLC margin (the distance between tumor and MLCs end). As the MLCs margin increase as the field size increase, so our study like study of effects of increasing field size on dose distribution within tumor, and all parameters which are affected by field size and contribute in dose distribution. The study is divided into two main parts. The first part includes the study of beam behavior with PDD, dose profile and penumbra for difference regular field size (with only by collimator jaws) with large increasing in field size, and then apply it to difference irregular field size (with MLCs) with small increasing in field size (the increasing rate is $0.1 \mathrm{~cm}$ ). The second part includes the study of effects of increasing field size on tumor and OARs for irregular fields using MLCs in shaping for pancreas cancer cases. There are many technique are used in pancreas cancer treatment, the most common three techniques were used in our study and the data of all were recorded. For all plans, the comparison held, and the best margins were chosen.

\section{Material and methods}

Materials: 10 patients during the period September 2012 to October 2013, Linear Accelerator DMX (Varian with photon energies 6 and 15 MV), Multi leaves Collimator (MLCs), Detectors (0.6 cc waterproof Farmer and 
semi - flex ionization chamber), water phantom (PTW - MP3-M water tank), dosimetry system with software (MEPHYSTO $\mathrm{mc}^{2}$ ), TANDEM Dual Channel Electrometer, Treatment planning System (Eclipse).

Method: two parts were done; the first part was measurements of PDD \& DP for regular and irregular fields and studies the effects of field size increasing on $\mathrm{D}_{\max }$, PDD, penumbra and opening collimator factor for dual energy $6 \& 15$ MV. The second part was 10 patients during the period September 2012 to October 2013; the cases were delineated carefully by doctors and the prescribed treatment dose was determined, then the three different treatment techniques plan were worked by the medical physicist and these plans were repeated with the only difference in MLC margin, the MLC margin started from $0.0 \mathrm{Cm}$ to $1.2 \mathrm{Cm}$, that is mean 39 plans were done for every patient. The percentage of dose that cover $95 \%$ of PTV volume and the mean dose for parallel OAR and point dose for serial OAR were taken and written in tables, three PTV cover curves in the dose percentage \& MLC margins relationship were plotted for every patient.

\section{Results}

The results were taken into two parts:

\section{Part 1 physical parameter:}

\section{Irregular field sizes were shaped by MLC.}

Measurements of beam profile for circular field size with radius $5.67 \mathrm{Cm}$ (equivalent field size to $10 \mathrm{x}$ $10 \mathrm{~cm}^{2}$ ) as example, with different beam energies (6MV and $15 \mathrm{MV} \mathrm{X}$ - rays) as show in Fig. 1.
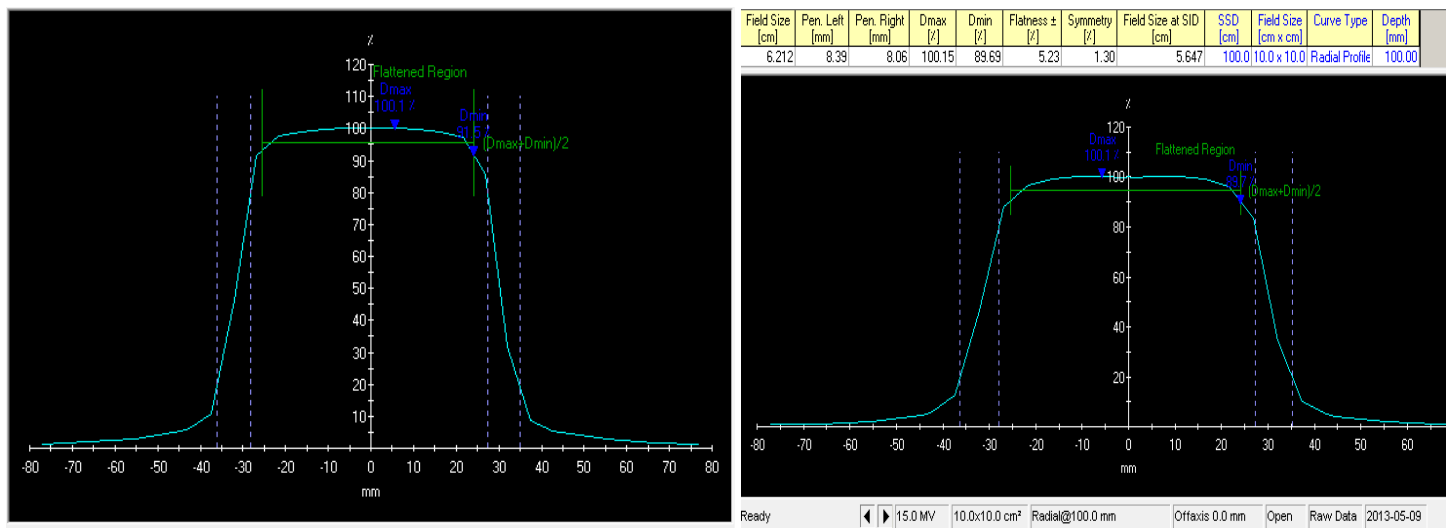

Fig. 1: the Beam Profile for field size $10 \times 10 \mathrm{~cm}^{2}$ and analysis for different parameters description for photon beam 6 \& $15 \mathrm{MV}$.

\begin{tabular}{|c|c|c|}
\hline \multirow{2}{*}{$\begin{array}{c}\text { MLC } \\
\text { margin }\end{array}$} & Energy 6 MV & Energy 15 MV \\
\cline { 2 - 3 } & Penumbra (mm) & Penumbra (mm) \\
\hline 0.0 Cm & 7.650 & 10.835 \\
$0.1 \mathrm{Cm}$ & 7.765 & 8.300 \\
$0.2 \mathrm{Cm}$ & 7.555 & 8.110 \\
0.3 Cm & 7.275 & 8.115 \\
0.4 Cm & 7.420 & 8.240 \\
$0.5 \mathrm{Cm}$ & 7.880 & 8.325 \\
$0.6 \mathrm{Cm}$ & 7.965 & 8.405 \\
$0.7 \mathrm{Cm}$ & 7.575 & 8.205 \\
$0.8 \mathrm{Cm}$ & 6.880 & 7.630 \\
$0.9 \mathrm{Cm}$ & 7.240 & 8.320 \\
$1.0 \mathrm{Cm}$ & 9.115 & 10.030 \\
$1.1 \mathrm{Cm}$ & 11.045 & 11.295 \\
$1.2 \mathrm{Cm}$ & 11.720 & 11.755 \\
\hline
\end{tabular}

Table 1: penumbra reading for dual energy $6 \& 15 \mathrm{MV}$ for different MLC margin for circular field sizes.

We note that the beam behavior for regular field size with relatively large increasing is the same for irregular circular field size with small increasing in field area "penumbra is sharper for higher energy and it is greater for high energy". 
Measuring Percent Depth Dose (PDD) curve.

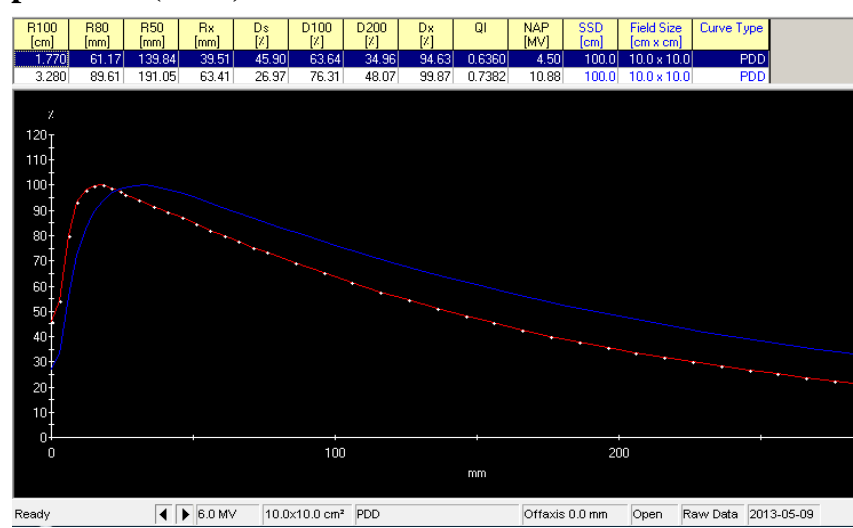

Fig. (2) Comparative PDD curves for 6 MV \&15 MV

\begin{tabular}{|l|l|l|l|l|l|l|l|}
\hline Beam & $0 \mathrm{~cm}$ & $\mathrm{Z}_{\text {max }}$ & $\begin{array}{l}\text { PDD at } \\
5 \mathrm{~cm}\end{array}$ & $\begin{array}{l}\text { PDD at } \\
10 \mathrm{~cm}\end{array}$ & PDD 20 & PDD 20/10 & $\begin{array}{l}\text { Beam } \\
\text { quality }\end{array}$ \\
\hline $6 \mathrm{MV}$ & 45.9 & $1.77 \mathrm{~cm}$ & $\mathbf{8 5 . 1 3 \%}$ & $\mathbf{6 3 . 6 4 \%}$ & $\mathbf{3 4 . 9 6 \%}$ & $\mathbf{0 . 5 4 9 3}$ & $\mathbf{0 . 6 3 6}$ \\
\hline $15 \mathrm{MV}$ & 26.97 & $\mathbf{3 . 2 8} \mathrm{cm}$ & $\mathbf{9 5 . 4 4 \%}$ & $\mathbf{7 6 . 3 1 \%}$ & $\mathbf{4 8 . 0 7 \%}$ & $\mathbf{0 . 6 2 9 9}$ & $\mathbf{0 . 7 3 8}$ \\
\hline
\end{tabular}

Table 2: analysis of PDD curves for $6 \& 15 \mathrm{MV}$ at different depths $\left(0, \mathrm{z}_{\max }, 5,10,20 \mathrm{Cm}\right)$

Effect of field size

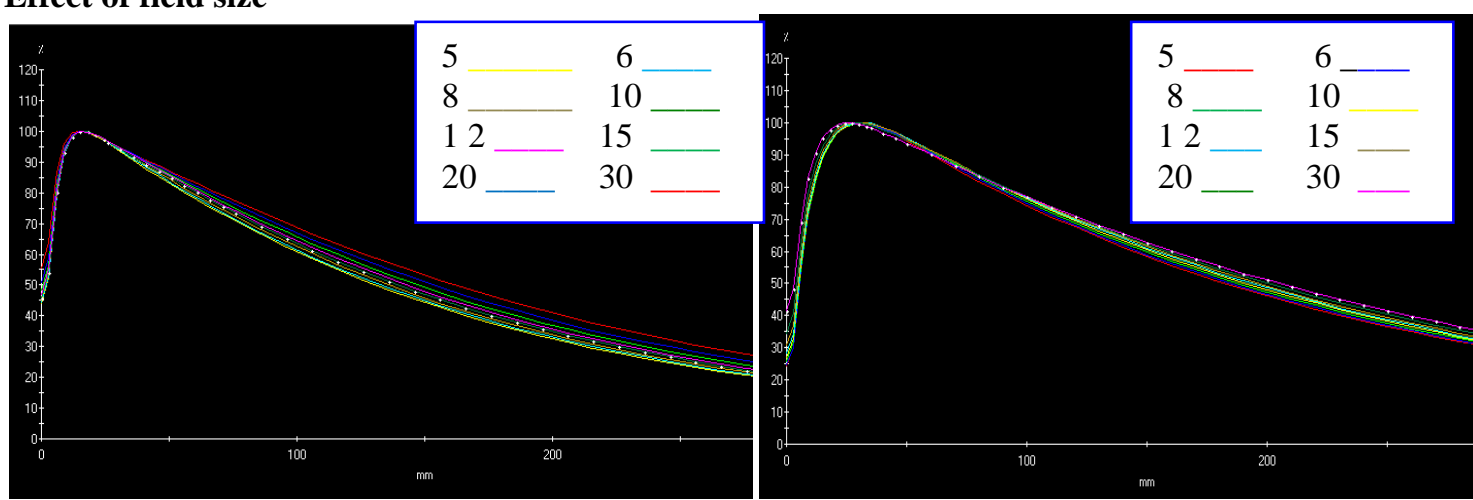

Fig (3) PDD curves for $6 \& 15 \mathrm{MV}$ photon beam at different field sizes

\begin{tabular}{|c|c|c|c|c|c|c|c|c|c|c|}
\hline F.S & Energy & PDD \% & & & Depth & & & & & \\
\hline equivalent & & $0 \mathrm{Cm}$ & $1 \mathrm{Cm}$ & $2 \mathrm{Cm}$ & $\mathbf{Z}_{\max }$ & $90 \%$ & $80 \%$ & $70 \%$ & $60 \%$ & $50 \%$ \\
\hline $5 \times 5$ & $6 \mathrm{MV}$ & 44.11 & 94.68 & 99.02 & 1.53 & 3.69 & 5.62 & 7.78 & 10.22 & 13.1 \\
\hline & $15 \mathrm{MV}$ & 24.47 & \begin{tabular}{|l|l|}
75.38 \\
\end{tabular} & 96.28 & 2.98 & 6.04 & 8.47 & 11.24 & 14.45 & 18.31 \\
\hline $6 \times 6$ & $6 \mathrm{MV}$ & 44.52 & 94.56 & 99.17 & 1.54 & 3.79 & 5.71 & 7.88 & 10.37 & 13.3 \\
\hline & $15 \mathrm{MV}$ & 24.65 & 74.67 & 95.98 & 3.01 & 6.14 & 8.64 & 11.42 & 14.64 & 18.5 \\
\hline $8 \times 8$ & $6 \mathrm{MV}$ & 45.28 & 94.69 & 99.14 & 1.54 & 3.88 & 5.9 & 8.13 & 10.68 & 13.6 \\
\hline & $15 \mathrm{MV}$ & 25.66 & 74.83 & 95.57 & 3.24 & 6.27 & 8.80 & 11.64 & 14.9 & 18.81 \\
\hline $10 \times 10$ & $6 \mathrm{MV}$ & 45.9 & 94.84 & 99.27 & 1.77 & 3.95 & 6.12 & 8.36 & 10.98 & 14 \\
\hline & $15 \mathrm{MV}$ & 26.97 & $\begin{array}{l}75.54 \\
\end{array}$ & \begin{tabular}{ll|}
95.8 \\
\end{tabular} & 3.28 & 6.34 & 8.96 & 11.82 & 15.2 & 19.1 \\
\hline $12 \times 12$ & $6 \mathrm{MV}$ & 46.88 & 95.06 & 99.13 & 1.54 & 4.05 & 6.23 & 8.55 & 11.21 & 14.3 \\
\hline & $15 \mathrm{MV}$ & 28.13 & 76.26 & 96.1 & 3.27 & 6.40 & 9.04 & 11.96 & 15.4 & 19.34 \\
\hline $15 \times 15$ & $6 \mathrm{MV}$ & 48.41 & 95.43 & 99.12 & 1.54 & 4.08 & 6.41 & 8.83 & 11.59 & 14.7 \\
\hline & $15 \mathrm{MV}$ & \begin{tabular}{|l|}
30.68 \\
\end{tabular} & $\begin{array}{l}78.1 \\
\end{array}$ & 96.78 & 3.02 & 6.38 & 9.10 & 12.11 & 15.6 & 19.63 \\
\hline $20 \times 20$ & $6 \mathrm{MV}$ & \begin{tabular}{|l|l|}
50.74 \\
\end{tabular} & 96.01 & 99.08 & 1.53 & 4.18 & 6.61 & 9.19 & 12.04 & 15.3 \\
\hline & $15 \mathrm{MV}$ & 34.50 & 80.69 & 97.68 & 2.77 & 6.26 & 9.11 & 12.26 & 15.8 & 20.05 \\
\hline $30 \times 30$ & $6 \mathrm{MV}$ & \begin{tabular}{|l|l|}
55.45 \\
\end{tabular} & 97.15 & 98.71 & 1.49 & 4.25 & 6.87 & 9.62 & 12.69 & 16.3 \\
\hline & $15 \mathrm{MV}$ & 41.48 & 85.33 & 98.84 & 2.46 & 6.03 & 9.00 & 12.33 & 16.1 & 20.51 \\
\hline
\end{tabular}

Table 3: measurements of PDD for different field size for 6 and $15 \mathrm{MV}$ 


\section{As field size increases:}

- $\quad \mathrm{Z}_{\max }$ decreases because of the increase scatter component make maximum dose point appears faster at less depth.

- $\quad$ PDD increases at other points along the central beam axis

- $\quad$ The ratio of the penumbra to the central portion of the field decreases

As field size decreases:

- $\quad \mathrm{Z}_{\max }$ increases because of the decrease scatter component make maximum dose point appears slower at greater depth.

- $\quad$ PDD decreases at points along the central beam axis

- The ratio of the penumbra to the central portion increases, and may lead to complete loss of the central flat section.

\section{Measuring beam profile curves}

Beam profile at gradual depths start from $\mathrm{d}_{\max }, 5 \mathrm{~cm}, 10 \mathrm{~cm}, 15 \mathrm{~cm}$ and $20 \mathrm{~cm}$ for 6 and $15 \mathrm{MV}$ were measured and analyzed as shown in figures $(23,24)$. From beam profile we can got to beam flatness, beam symmetry and penumbra
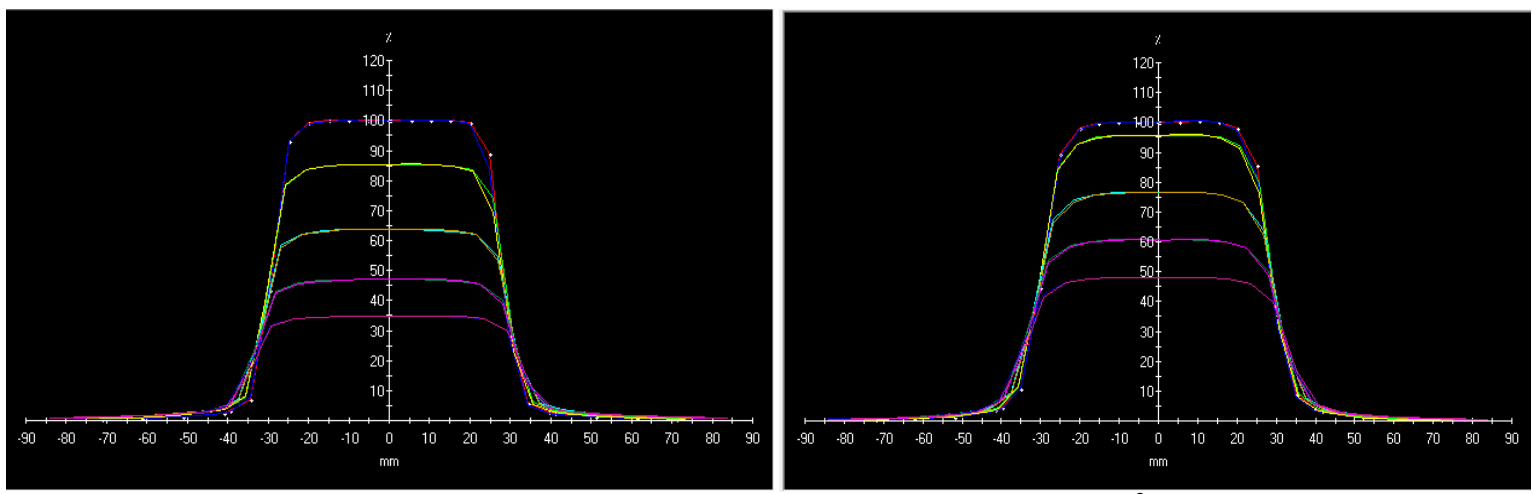

fig(4) $6 \& 15 \mathrm{MV}$ beam profile, field size $10 \times 10 \mathrm{Cm}^{2}$.

\begin{tabular}{|c|c|c|c|c|c|c|c|}
\hline & & \multicolumn{3}{|c|}{ Radial profile } & \multicolumn{3}{|c|}{ Transverse profile } \\
\hline Depths & Energy & Pen. (mm) & F. \% & S. \% & Pen. (mm) & F. \% & S. \% \\
\hline \multirow{2}{*}{$\mathbf{D}_{\max }$} & $6 \mathrm{MV}$ & 6.55 & 3.36 & 0.2 & 6.52 & 4.09 & 0.24 \\
\hline & $15 \mathrm{MV}$ & 7.40 & 4.54 & 1.24 & 7.44 & 5.28 & 1.46 \\
\hline \multirow[t]{2}{*}{$5 \mathrm{~cm}$} & $6 \mathrm{MV}$ & 7.09 & 4.09 & 0.25 & 6.97 & 4.86 & $\mathbf{0 . 3 8}$ \\
\hline & $15 \mathrm{MV}$ & 7.73 & 5.07 & 1.30 & 7.87 & 6.03 & 2.11 \\
\hline \multirow[t]{2}{*}{$10 \mathrm{~cm}$} & $6 \mathrm{MV}$ & 7.66 & 4.45 & 0.4 & 7.66 & 4.90 & 0.18 \\
\hline & $15 \mathrm{MV}$ & 8.23 & 5.23 & 1.30 & 8.28 & 5.75 & 1.45 \\
\hline \multirow[t]{2}{*}{$15 \mathrm{~cm}$} & $6 \mathrm{MV}$ & 8.09 & 4.48 & 0.69 & 8.07 & 4.99 & 0.55 \\
\hline & $15 \mathrm{MV}$ & 8.64 & 5.34 & 1.58 & 8.65 & 5.86 & 1.65 \\
\hline \multirow[t]{2}{*}{$20 \mathrm{~cm}$} & $6 \mathrm{MV}$ & 8.55 & 4.67 & 0.82 & 8.62 & 4.66 & 0.45 \\
\hline & $15 \mathrm{MV}$ & 9.04 & 5.31 & 1.72 & 9.14 & 5.61 & 0.98 \\
\hline
\end{tabular}

Table 4: penumbra, flatness and symmetry for radial and transverse profile measured for 6 and $15 \mathrm{MV}$ energy. Determination of the Opening Collimator Factor (OCF)

The ratio between the maximal absorbed dose and the absorbed dose in the reference depth at depth 10 $\mathrm{cm}$ were calculated, and then we make varied the field size. Theses curves are normalized to cyclic field size equivalent to $10 \mathrm{~cm} \times 10 \mathrm{~cm}$ field size. The $\mathrm{OFC}$ increases with the field size. So, the dose in the measured point increases with the field size. This increasing is more important for higher photon beam energy and for dose distribution.

\begin{tabular}{|c|c|c|}
\hline \multirow{2}{*}{ Filed size } & \multicolumn{2}{|c|}{ OCF } \\
\cline { 2 - 3 } & $6 \mathrm{MV}$ & $15 \mathrm{MV}$ \\
\hline $5 \times 5$ & 0.956 & 0.971 \\
\hline $6 \times 6$ & 0.965 & 0.979 \\
& & 0.990 \\
\hline $8 \times 8$ & 0.983 & 1 \\
\hline $10 \times 10$ & 1.000 & 1.003 \\
\hline $15 \times 12$ & 1.010 & 1.007 \\
\hline $20 \times 20$ & 1.031 & 1.008 \\
\hline $30 \times 30$ & 1.053 & 1.010 \\
\hline
\end{tabular}

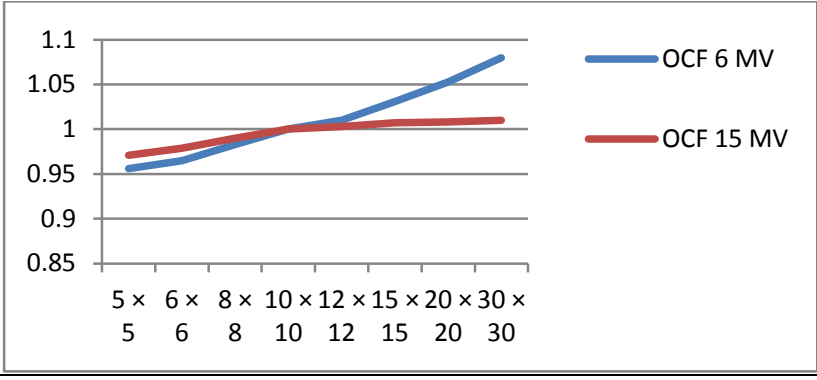

Table 5: opening collimator factor for different field size, for 6 and $15 \mathrm{MV} \&$ diagram of the reading. 
Part 2 Optimization of Conformal Radiotherapy for pancreas cancer patients:-

To illustrate the impact of expansion MLCs margin around the target in conformal 3D treatment planning real patient situations. 13 different plans were generated for 10 patients using the same arrangements and the only different is the MLC settings. A dose of $5040 \mathrm{cGy}$ in 28 fractions was prescribed and normalized to $100 \%$ at the mean of target defined. The result unloaded in the following tables.

The case No. 1

A middle age woman (42 y.) with pancreas cancer,

\begin{tabular}{|c|c|c|c|c|c|c|c|c|c|c|c|c|c|c|}
\hline Plan & MLC & 0.0 & 0.1 & 0.2 & 0.3 & 0.4 & 0.5 & 0.6 & 0.7 & 0.8 & 0.9 & 1.0 & 1.1 & 1.2 \\
\hline \multirow{6}{*}{$\begin{array}{l}3 \\
\text { Fields }\end{array}$} & PTV & 87.83 & 90.32 & 92.06 & 93.37 & 94.68 & 95.60 & 96.37 & 96.90 & 97.20 & 97.54 & 97.76 & 97.92 & 98.13 \\
\hline & S. Cord & 1575 & 1577 & 1582 & 1590 & 1597 & 1610 & 1627 & 1650 & 1710 & 1750 & 1800 & 1850 & 1880 \\
\hline & Lt.kid & 380 & 420 & 460 & 500 & 550 & 600 & 650 & 710 & 770 & 840 & 920 & 1000 & 1070 \\
\hline & Rt.kid & 1180 & 1240 & 1310 & 1370 & 1450 & 1530 & 1610 & 1700 & 1800 & 1900 & 2000 & 2110 & 2200 \\
\hline & Liver & 1060 & 1100 & 1140 & 1200 & 1250 & 1310 & 1370 & 1440 & 1500 & 1560 & 1630 & 1690 & 1750 \\
\hline & Max.Dose & 105.6 & 104.9 & 104.4 & 104.5 & 104.7 & 105 & 105.2 & 105.4 & 105.6 & 105.8 & 105.9 & 106 & 106.2 \\
\hline \multirow{6}{*}{$\begin{array}{l}4 \\
\text { Fields }\end{array}$} & PTV & 87.46 & 90.14 & 91.39 & 93.39 & 94.46 & 95.67 & 96.31 & 96.85 & 97.16 & 97.46 & 97.67 & 97.86 & 98.00 \\
\hline & S. Cord & 2712 & 2602 & 2604 & 2598 & 2597 & 2600 & 2608 & 2621 & 2636 & 2656 & 2679 & 2710 & 2753 \\
\hline & Lt.kid & 290 & 390 & 410 & 460 & 510 & 550 & 600 & 660 & 720 & 780 & 850 & 920 & 1000 \\
\hline & Rt.kid & 1490 & 1590 & 1630 & 1720 & 1800 & 1880 & 1970 & 2070 & 2170 & 2280 & 2380 & 2490 & 2590 \\
\hline & Liver & 880 & 990 & 1010 & 1050 & 1110 & 1190 & 1240 & 1310 & 1370 & 1430 & 1490 & 1550 & 1600 \\
\hline & Max Dose & 106.3 & 106 & 105.5 & 105.1 & 104.8 & 104.6 & 104.4 & 104.3 & 104.3 & 104.4 & 104.4 & 104.6 & 104.6 \\
\hline \multirow{6}{*}{$\begin{array}{l}5 \\
\text { Fields }\end{array}$} & PTV & 87.67 & 90.28 & 92.04 & 93.43 & 94.6 & 95.55 & 96.33 & 96.74 & 97.14 & 97.44 & 97.66 & 97.78 & 97.92 \\
\hline & S. Cord & 2090 & 2120 & 2150 & 2170 & 2170 & 2200 & 2210 & 2230 & 2250 & 2270 & 2320 & 2350 & 2430 \\
\hline & Lt.kid & 550 & 580 & 610 & 660 & 700 & 780 & 820 & 870 & 920 & 960 & 1030 & 1080 & 1140 \\
\hline & Rt.kid & 1250 & 1310 & 1360 & 1420 & 1490 & 1610 & 1710 & 1790 & 1880 & 1970 & 2060 & 2150 & 2230 \\
\hline & Liver & 960 & 1000 & 1040 & 1090 & 1140 & 1210 & 1260 & 1340 & 1400 & 1450 & 1510 & 1570 & 1620 \\
\hline & Max Dose & 106.6 & 105.9 & 105.4 & 105.1 & 104.9 & 104.7 & 104.6 & 104.5 & 104.5 & $\begin{array}{l}104.4 \\
\end{array}$ & 104.6 & 104.7 & 104.7 \\
\hline
\end{tabular}

Table 6: a table illustrates all OAR value and PTV volume percentage cover for three different techniques with

MLC difference margin start from $(0.0 \mathrm{~cm}$ to $1.2 \mathrm{~cm})$.

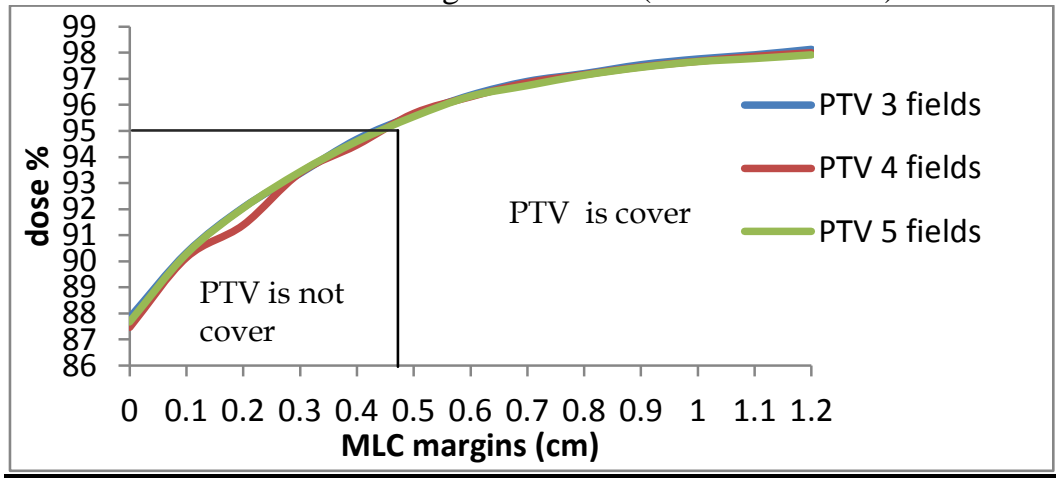

Fig. (5) PTV \& MLC margins.

The case No. 2

A woman (56 y.) with pancreas cancer 


\begin{tabular}{|c|c|c|c|c|c|c|c|c|c|c|c|c|c|c|}
\hline Plan & MLC & 0.0 & 0.1 & 0.2 & 0.3 & 0.4 & 0.5 & 0.6 & 0.7 & 0.8 & 0.9 & 1.0 & 1.1 & 1.2 \\
\hline \multirow{6}{*}{$\begin{array}{l}3 \\
\text { Fields }\end{array}$} & PTV & 87.26 & 90.32 & 93 & 94.5 & 95.3 & 96.3 & 96.8 & 97.3 & 97.4 & 97.6 & 97.66 & 97.66 & 97.66 \\
\hline & S. Cord & 1936 & 1940 & 1947 & 1954 & 1960 & 1980 & 2050 & 2180 & 2950 & 3774 & 1015 & 4160 & 4226 \\
\hline & Lt.kid & 1357 & 1424 & 1495 & 1561 & 1628 & 1744 & 1840 & 1969 & 2156 & 2323 & 2437 & 2530 & 2618 \\
\hline & Rt.kid & 1886 & 1950 & 2023 & 2091 & 2160 & 2280 & 2380 & 2520 & 2700 & 2892 & 3019 & 3110 & 3195 \\
\hline & Liver & 2051 & 2116 & 2214 & 2305 & 2379 & 2500 & 2575 & 2670 & 2755 & 2838 & 2922 & 2992 & 3089 \\
\hline & Max.Dose & 104.5 & 104.3 & 103.8 & 103.4 & 103.2 & 103 & 102.9 & 103 & 102.9 & 102.9 & 102.9 & 103 & 103.2 \\
\hline \multirow{6}{*}{$\begin{array}{l}4 \\
\text { Fields }\end{array}$} & PTV & 87.26 & 90.32 & 93 & 94.5 & 95.3 & 96.3 & 96.8 & 97.3 & 97.4 & 97.6 & 97.66 & 97.66 & 97.66 \\
\hline & S. Cord & 3043 & 3029 & 3022 & 3019 & 3025 & 3029 & 3055 & 3132 & 3636 & 4158 & 4336 & 4429 & 4469 \\
\hline & Lt.kid & 1301 & 1382 & 1462 & 1538 & 1614 & 1721 & 1813 & 1930 & 2085 & 2226 & 2333 & 2429 & 2518 \\
\hline & Rt.kid & 2122 & 2206 & 2291 & 2372 & 2455 & 2566 & 2674 & 2814 & 2968 & 3125 & 3241 & 3331 & 3417 \\
\hline & Liver & 1830 & 1893 & 1982 & 2063 & 2136 & 2239 & 2310 & 2398 & 2479 & 2557 & 2638 & 2710 & 2800 \\
\hline & Max.Dose & 105.5 & 104.7 & 104.1 & 103.6 & 103.3 & 103 & 102.8 & 102.7 & 102.6 & 102.5 & 102.4 & 102.4 & 102.3 \\
\hline \multirow{6}{*}{$\begin{array}{l}5 \\
\text { Fields }\end{array}$} & PTV & 87.54 & 90.08 & 92.52 & 93.95 & 94.74 & 95.75 & 96.47 & 96.96 & 97.18 & 97.32 & 97.40 & 97.44 & 97.52 \\
\hline & S. Cord & 2740 & 2732 & 2729 & 2729 & 2730 & 2746 & 2776 & 2867 & 3439 & 4034 & 4216 & 4322 & 4371 \\
\hline & Lt.kid & 1402 & 1464 & 1531 & 1593 & 1653 & 1750 & 1836 & 1944 & 2093 & 2261 & 2364 & 2448 & 2530 \\
\hline & Rt.kid & 1987 & 2051 & 2123 & 2193 & 2264 & 2375 & 2479 & 2607 & 2772 & 2929 & 3046 & 3130 & 3216 \\
\hline & Liver & 1945 & 2028 & 2113 & 2192 & 2265 & 2368 & 2444 & 2531 & 2614 & 2691 & 2771 & 2861 & 2949 \\
\hline & Max.Dose & 105.4 & 104.6 & 104 & 103.6 & 103.4 & 103. & 103.1 & 103.1 & 103 & 103 & 103 & 103.1 & 103.1 \\
\hline
\end{tabular}

Table 7: a table illustrates all OAR value and PTV volume percentage cover for three different techniques with MLC difference margin start from $(0.0 \mathrm{~cm}$ to $1.2 \mathrm{~cm})$.

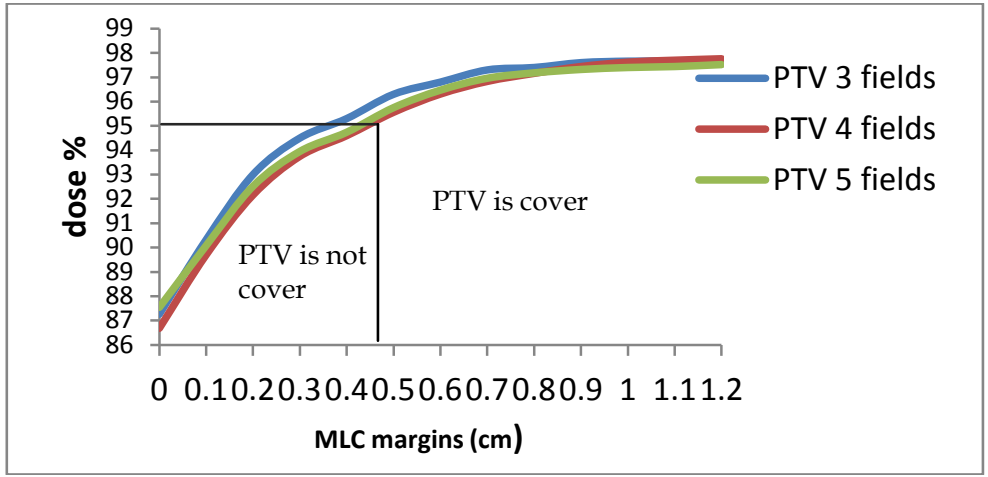

Fig. (6) PTV \& MLC margins.

The case No. 3

A woman (38 y.) with pancreas cancer,

\begin{tabular}{|c|c|c|c|c|c|c|c|c|c|c|c|c|c|c|}
\hline Plan & MLC & 0.0 & 0.1 & 0.2 & 0.3 & 0.4 & 0.5 & 0.6 & 0.7 & 0.8 & 0.9 & 1.0 & 1.1 & 1.2 \\
\hline \multirow{6}{*}{$\begin{array}{l}3 \\
\text { Fields }\end{array}$} & PTV & 88.37 & 91.0 & 92.40 & 93.40 & 94.05 & 94.58 & 95.14 & 95.54 & 95.87 & 96.11 & 96.29 & 96.47 & 96.63 \\
\hline & S. Cord & 1714 & 1746 & 1801 & 1848 & 1882 & 1918 & 1989 & 2046 & 2120 & 2170 & 2218 & 2396 & 2680 \\
\hline & $\begin{array}{l}\text { Lt.kid } \\
\end{array}$ & 1361 & 1400 & 1430 & 1480 & 1508 & 1620 & 1700 & 1741 & 1800 & 1846 & 1910 & 1967 & 2021 \\
\hline & Rt.kid & 1400 & 1455 & 1508 & 1595 & 1643 & 1819 & 1922 & 1982 & 2055 & 2111 & 2211 & 2280 & 2345 \\
\hline & Liver & 1280 & 1341 & 1393 & 1448 & 1493 & 1561 & 1627 & 1684 & 1751 & 1810 & 1882 & 1958 & 2024 \\
\hline & Max.Dose & 105.1 & 104.6 & 104.3 & 104.3 & 104.6 & 104.9 & 104.9 & 104.9 & 104.9 & 104.9 & 105 & 105 & 105.1 \\
\hline \multirow{6}{*}{$\begin{array}{l}4 \\
\text { Fields }\end{array}$} & PTV & 88.21 & 90.85 & 92.34 & 93.43 & 94.07 & 94.70 & 95.08 & 95.42 & 95.73 & 95.91 & 96.03 & 96.11 & 96.15 \\
\hline & S. Cord & 2920 & 2909 & 2907 & 2919 & 2930 & 2985 & 3041 & 3079 & 3130 & 3160 & 3179 & 3234 & 3526 \\
\hline & Lt.kid & 2035 & 2090 & 2138 & 2200 & 2243 & 2378 & 2413 & 2494 & 2521 & 2569 & 2634 & 2697 & 2838 \\
\hline & Rt.kid & 1851 & 1928 & 2000 & 2096 & 2163 & 2362 & 2419 & 2460 & 2578 & 2646 & 2745 & 2819 & 2988 \\
\hline & Liver & 1071 & 1128 & 1180 & 1232 & 1275 & 1351 & 1395 & 1452 & 1516 & 1575 & 1641 & 1714 & 1787 \\
\hline & Max.Dose & 105.3 & 104.7 & 104.4 & 104.2 & 104.1 & 103.9 & 103.8 & 103.8 & 103.7 & 103.7 & 103.6 & 103.6 & 105.3 \\
\hline \multirow{6}{*}{$\begin{array}{l}5 \\
\text { Fields }\end{array}$} & PTV & 88.93 & 90.81 & 92.40 & 93.31 & 94.07 & 94.64 & 95.14 & 95.58 & 95.91 & 96.13 & 96.23 & 96.37 & 96.47 \\
\hline & S. Cord & 2390 & 2395 & 2431 & 2449 & 2494 & 2578 & 2599 & 2640 & 2666 & 2726 & 2840 & 2919 & 3009 \\
\hline & Lt.kid & 1543 & 1580 & 1672 & 1720 & 1779 & 1850 & 1886 & 1929 & 1973 & 2028 & 2118 & 2166 & 2309 \\
\hline & Rt.kid & 1568 & 1612 & 1738 & 1791 & 1866 & 1940 & 1986 & 2051 & 2123 & 2203 & 2312 & 2362 & 2533 \\
\hline & Liver & 1198 & 1248 & 1313 & 1361 & 1416 & 1475 & 1532 & 1598 & 1659 & 1722 & 1796 & 1861 & 1939 \\
\hline & Max.Dose & 105.2 & 104.7 & 104.2 & 104 & 103.9 & 103.9 & 103.9 & 103.9 & 103.9 & 104 & 104.4 & 104.6 & 104.7 \\
\hline
\end{tabular}

Table 8: a table illustrates all OAR value and PTV volume percentage cover for three different techniques with

MLC difference margin start from $(0.0 \mathrm{~cm}$ to $1.2 \mathrm{~cm})$. 


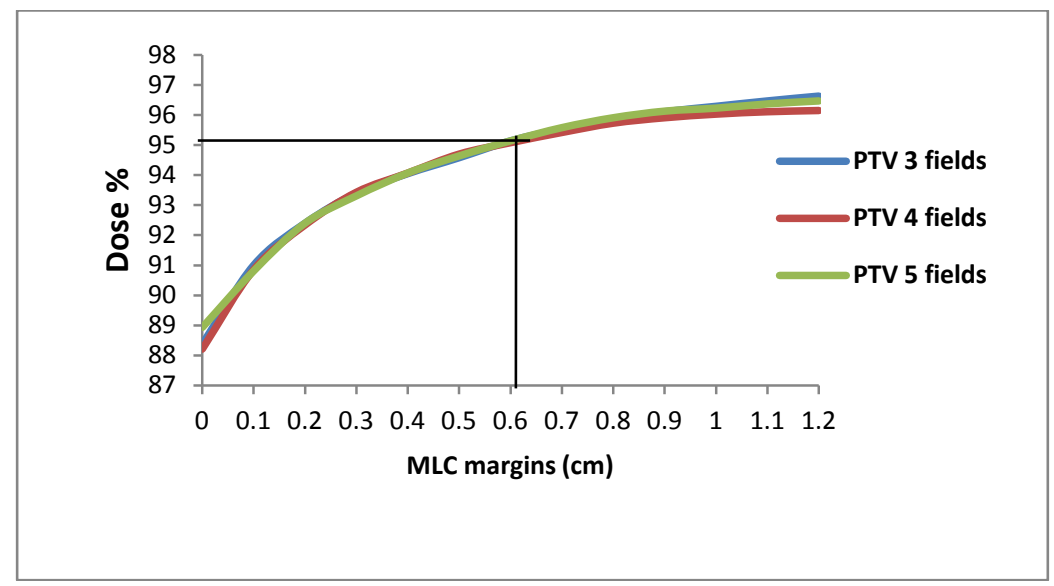

Fig. (7) PTV \& MLC margins.

The case No. 4

A man (56 y.) with pancreas cancer,

\begin{tabular}{|c|c|c|c|c|c|c|c|c|c|c|c|c|c|c|}
\hline Plan & MLC & 0.0 & 0.1 & 0.2 & 0.3 & 0.4 & 0.5 & 0.6 & 0.7 & 0.8 & 0.9 & 1.0 & 1.1 & 1.2 \\
\hline \multirow{6}{*}{$\begin{array}{l}3 \\
\text { Fields }\end{array}$} & PTV & 87.10 & 90.40 & 92.72 & 94.11 & 95.34 & 96.03 & 96.55 & 97.06 & 97.42 & 97.70 & 97.88 & 97.94 & 97.99 \\
\hline & S. Cord & 1589 & 1584 & 1585 & 1587 & 1591 & 1595 & 1599 & 1605 & 1611 & 1619 & 1628 & 1640 & 1650 \\
\hline & Lt.kid & 467 & 529 & 586 & 623 & 665 & 705 & 742 & 794 & 841 & 948 & 1046 & 1153 & 1243 \\
\hline & Rt.kid & 349 & 374 & 405 & 423 & 459 & 486 & 518 & 550 & 579 & 620 & 663 & 721 & 775 \\
\hline & Liver & 2285 & 2339 & 2385 & 2423 & 2486 & 2539 & 2602 & 2662 & 2706 & 2794 & 2826 & 2861 & 2978 \\
\hline & Max.Dose & 105.7 & 104.9 & 104.4 & 104.1 & 103.7 & 103.6 & 103.4 & 103.3 & 103.2 & 103.4 & 103.6 & 103.7 & 103.8 \\
\hline \multirow{6}{*}{$\begin{array}{l}4 \\
\text { Fields }\end{array}$} & PTV & 87.12 & 90.50 & 92.82 & 94.27 & 95.48 & 96.31 & 96.81 & 97.30 & 97.62 & 97.84 & 97.96 & 98.02 & 98.08 \\
\hline & S. Cord & 2903 & 2881 & 2865 & 2855 & 2846 & 2842 & 2836 & 2833 & 2834 & 2833 & 2836 & 2841 & 2845 \\
\hline & Lt.kid & 507 & 566 & 622 & 662 & 704 & 746 & 783 & 832 & 879 & 967 & 1053 & 1145 & 1228 \\
\hline & Rt.kid & 407 & 437 & 472 & 505 & 539 & 575 & 613 & 651 & 691 & 736 & 786 & 844 & 903 \\
\hline & Liver & 1900 & 1948 & 1991 & 2030 & 2088 & 2138 & 2195 & 2250 & 2294 & 2368 & 2428 & 2486 & 2535 \\
\hline & Max Dose & 105 & 104.2 & 103.6 & $\begin{array}{l}103.3 \\
\end{array}$ & 103.1 & 102.9 & 102.9 & 102.8 & 102.7 & 102.6 & 102.7 & 102.7 & 102.8 \\
\hline \multirow{6}{*}{$\begin{array}{l}5 \\
\text { Fields }\end{array}$} & PTV & 87.70 & 90.80 & 92.84 & 94.17 & 95.18 & 95.83 & 96.27 & 96.79 & 97.06 & 97.32 & 97.48 & 97.58 & 97.68 \\
\hline & S. Cord & 1744 & 1784 & 1843 & 1884 & 1929 & 1955 & 1980 & 2001 & 2020 & 2040 & 2057 & 2071 & 2086 \\
\hline & Lt.kid & 587 & 641 & 690 & 725 & 763 & 799 & 835 & 882 & 925 & 1018 & 1104 & 1197 & 1276 \\
\hline & Rt.kid & 506 & 528 & 556 & 578 & 604 & 628 & 657 & 685 & 711 & 747 & 785 & 836 & 882 \\
\hline & Liver & 2180 & 2239 & 2288 & 2326 & 2391 & 2441 & 2500 & 2558 & 2601 & 2687 & 2750 & 2817 & 2875 \\
\hline & Max Dose & 105.2 & 104.4 & 103.9 & 103.6 & 103.4 & 103.3 & 103.2 & 103.1 & 103 & 103 & 103 & 103.1 & 103.3 \\
\hline
\end{tabular}

Table 9: a table illustrates all OAR value and PTV volume percentage cover for three different techniques with

MLC difference margin start from $(0.0 \mathrm{~cm}$ to $1.2 \mathrm{~cm})$.

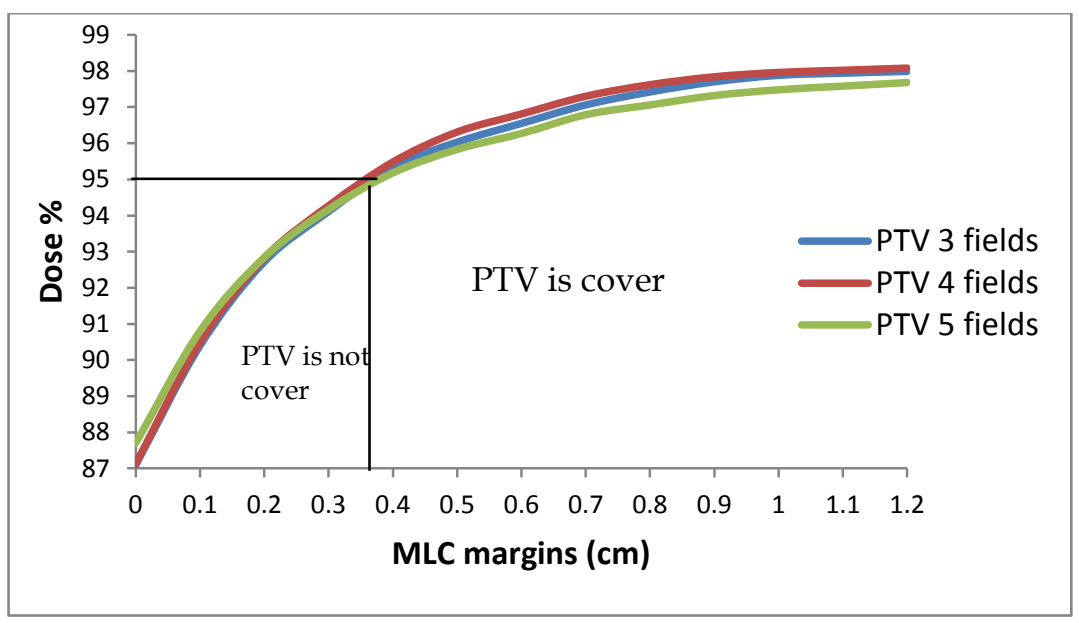

The case No. 5

Fig. (8) PTV \& MLC margins.

A man (52 y.) with pancreas cancer, 


\begin{tabular}{|c|c|c|c|c|c|c|c|c|c|c|c|c|c|c|}
\hline Plan & MLC & 0.0 & 0.1 & 0.2 & 0.3 & 0.4 & 0.5 & 0.6 & 0.7 & 0.8 & 0.9 & 1.0 & 1.1 & 1.2 \\
\hline \multirow{6}{*}{$\begin{array}{l}3 \\
\text { Fields }\end{array}$} & PTV & 83.83 & 88.08 & 91.39 & 93.73 & 95.14 & 96.00 & 96.70 & 96.98 & 97.54 & 97.84 & 98.06 & 98.25 & 98.39 \\
\hline & S. Cord & 1986 & 1982 & 1995 & 2005 & 2011 & 2113 & 2159 & 2214 & 2399 & 2573 & 2887 & 3055 & 3657 \\
\hline & Lt.kid & 340 & 380 & 433 & 474 & 523 & 606 & 653 & 698 & 756 & 811 & 873 & 937 & 1009 \\
\hline & Rt.kid & 172 & 192 & 215 & 242 & 266 & 298 & 331 & 359 & 390 & 420 & 451 & 486 & 521 \\
\hline & Liver & 877 & 921 & 961 & 1008 & 1050 & 1093 & 1141 & 1187 & 1235 & 1281 & 1325 & 1379 & 1430 \\
\hline & Max.Dose & 106.4 & 105.3 & 104.4 & 103.8 & 103.5 & 103.2 & 102.9 & 102.8 & 102.8 & 102.8 & 102.8 & 102.8 & 102.9 \\
\hline \multirow{5}{*}{$\begin{array}{l}4 \\
\text { Fields }\end{array}$} & PTV & 83.73 & 88.23 & 91.47 & 93.71 & 95.24 & 96.15 & 96.83 & 97.12 & 97.70 & 98.02 & 98.21 & 98.41 & 98.53 \\
\hline & Lt.kid & 278 & 310 & 353 & 387 & 429 & 493 & 532 & 570 & 619 & 666 & 720 & 775 & 838 \\
\hline & Rt.kid & 149 & 165 & 185 & 208 & 230 & 257 & 286 & 310 & 338 & 365 & 392 & 426 & 454 \\
\hline & Liver & 774 & 813 & 850 & 890 & 926 & 966 & 1010 & 1051 & 1096 & 1138 & 1178 & 1229 & 1276 \\
\hline & Max Dose & 106.6 & 105.4 & 104.5 & 103.9 & 103.4 & 103.2 & 103 & 103 & 102.9 & 102.9 & 103 & 102.9 & 103 \\
\hline \multirow{5}{*}{$\begin{array}{l}5 \\
\text { Fields }\end{array}$} & PTV & 85.97 & 89.11 & 91.85 & 94.01 & 95.38 & 96.11 & 96.75 & 97.22 & 97.54 & 97.82 & 98.02 & 98.60 & 98.27 \\
\hline & S. Cord & 2358 & 2392 & 2404 & 2425 & 2431 & 2483 & 2514 & 2635 & 2789 & 2939 & 3210 & 3358 & 3880 \\
\hline & Lt.kid & 441 & 480 & 529 & 567 & 614 & 685 & 730 & 771 & 825 & 876 & 931 & 990 & 1054 \\
\hline & Liver & 870 & 911 & 955 & 1001 & 1044 & 1087 & 1136 & 1182 & 1232 & 1279 & 1324 & 1380 & 1432 \\
\hline & Max Dose & 106.3 & 105.1 & 104.3 & 103.6 & 103.3 & 103.1 & 102.8 & 102.7 & 102.7 & 102.7 & 102.8 & 102.9 & 102.9 \\
\hline
\end{tabular}

Table 10: a table illustrates all OAR value and PTV volume percentage cover for three different techniques with MLC difference margin start from $(0.0 \mathrm{~cm}$ to $1.2 \mathrm{~cm})$.

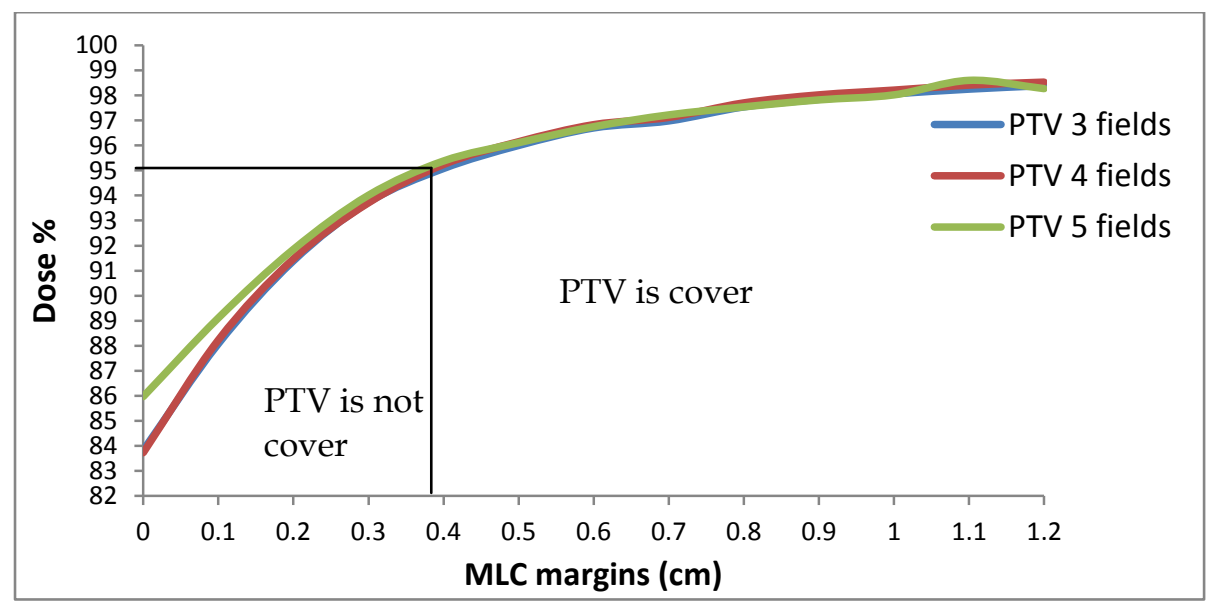

The case No. 6

Fig. (9) PTV \& MLC margins.

A man (58 y.) with pancreas cancer,

\begin{tabular}{|c|c|c|c|c|c|c|c|c|c|c|c|c|c|c|}
\hline Plan & MLC & 0.0 & 0.1 & 0.2 & 0.3 & 0.4 & 0.5 & 0.6 & 0.7 & 0.8 & 0.9 & 1.0 & 1.1 & 1.2 \\
\hline \multirow{6}{*}{$\begin{array}{l}3 \\
\text { Fields }\end{array}$} & PTV & 85.97 & 89.11 & 91.85 & 94.01 & 95.38 & 96.11 & 96.75 & 97.22 & 97.54 & 97.82 & 98.02 & 98.60 & 98.27 \\
\hline & S. Cord & 1562 & 1618 & 1708 & 1905 & 2000 & 2020 & 2251 & 2467 & 2683 & 2843 & 2917 & 3041 & 3017 \\
\hline & Lt.kid & 919 & 977 & 1026 & 1083 & 1137 & 1194 & 1265 & 1327 & 1393 & 1468 & 1535 & 1622 & 1703 \\
\hline & Rt.kid & 190 & 208 & 226 & 242 & 261 & 291 & 326 & 352 & 380 & 408 & 437 & 469 & 504 \\
\hline & Liver & 2232 & 2300 & 2370 & 2440 & 2506 & 2574 & 2644 & 2705 & 2772 & 2834 & 2896 & 2956 & 3017 \\
\hline & Max.Dose & 106.5 & 106.2 & 105.8 & 105.7 & 105.5 & 105.3 & 105.2 & 105.1 & 105 & 105.1 & 105.1 & 105.1 & 105.2 \\
\hline \multirow{6}{*}{$\begin{array}{l}4 \\
\text { Fields }\end{array}$} & PTV & 86.96 & 90.05 & 92.12 & 93.39 & 94.36 & 95.23 & 95.75 & 96.07 & 96.41 & 96.65 & 96.72 & 96.76 & 96.78 \\
\hline & S. Cord & 2370 & 2411 & 2484 & 2636 & 2710 & 2726 & 2901 & 3068 & 3236 & 3358 & 3423 & 3522 & 3651 \\
\hline & Lt.kid & 1228 & 1294 & 1348 & 1410 & 1470 & 1533 & 1610 & 1676 & 1742 & 1817 & 1886 & 1974 & 2054 \\
\hline & Rt.kid & 186 & 204 & 221 & 237 & 255 & 285 & 318 & 344 & 372 & 401 & 430 & 463 & 497 \\
\hline & Liver & 1905 & 1967 & 2035 & 2099 & 2161 & 2227 & 2293 & 2351 & 2415 & 2476 & 2537 & 2596 & 2634 \\
\hline & Max Dose & 105.3 & 105 & 104.9 & 104.8 & 104.7 & 104.5 & 104.5 & 104.4 & 104.3 & 104.3 & 104.3 & 104.3 & 104.6 \\
\hline \multirow{6}{*}{$\begin{array}{l}5 \\
\text { Fields }\end{array}$} & PTV & 86.66 & 89.66 & 91.74 & 92.95 & 93.86 & 94.46 & 94.92 & 95.23 & 95.39 & 95.55 & 95.59 & 95.67 & 95.89 \\
\hline & S. Cord & 1659 & 1693 & 1782 & 1946 & 2026 & 2051 & 2240 & 2421 & 2620 & 2808 & 2964 & 3138 & 3243 \\
\hline & Lt.kid & 1085 & 1150 & 1206 & 1270 & 1329 & 1393 & 1469 & 1535 & 1606 & 1685 & 1756 & 1845 & 1928 \\
\hline & Rt.kid & 255 & 274 & 293 & 310 & 330 & 360 & 393 & 419 & 447 & 476 & 504 & 538 & 572 \\
\hline & Liver & 2023 & 2086 & 2153 & 2226 & 2288 & 2357 & 2430 & 2490 & 2556 & 2618 & 2682 & 2742 & 2801 \\
\hline & Max Dose & 105.1 & 104.9 & 104.7 & 104 & 104.8 & 104.8 & 104.8 & 104.7 & 105 & 105.1 & 105.2 & 105.3 & 105.4 \\
\hline
\end{tabular}

Table 11: a table illustrates all OAR value and PTV volume percentage cover for three different techniques with MLC difference margin start from $(0.0 \mathrm{~cm}$ to $1.2 \mathrm{~cm})$. 


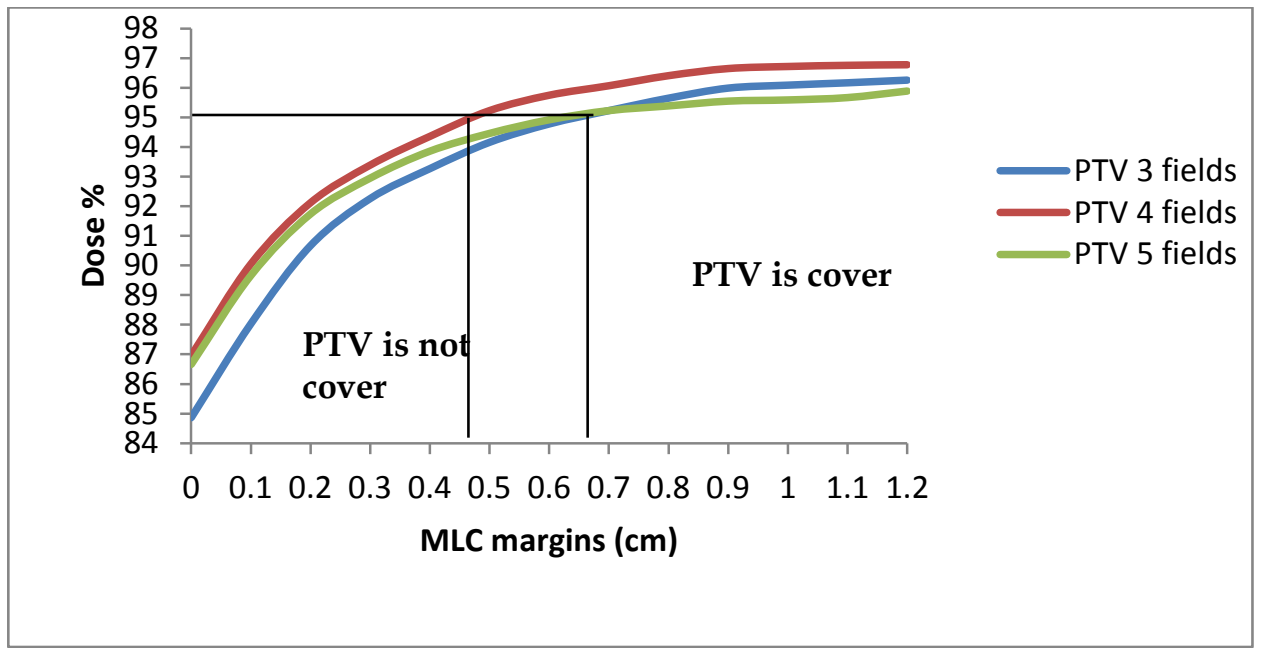

Fig. (10) PTV \& MLC margins.

The case No. 7

A man $(51 \mathrm{y}$.) with pancreas cancer,

Table 12: a table illustrates all OAR value and PTV volume percentage cover for three different techniques with

\begin{tabular}{|c|c|c|c|c|c|c|c|c|c|c|c|c|c|c|}
\hline Plan & MLC & 0.0 & 0.1 & 0.2 & 0.3 & 0.4 & 0.5 & 0.6 & 0.7 & 0.8 & 0.9 & 1.0 & 1.1 & 1.2 \\
\hline \multirow{6}{*}{$\begin{array}{l}3 \\
\text { Fields }\end{array}$} & PTV & 86.90 & 89.82 & 91.64 & 93.03 & 93.94 & 95.00 & 95.63 & 96.03 & 96.32 & 96.46 & 96.68 & 96.82 & 96.86 \\
\hline & S. Cord & 1520 & 1517 & 1524 & 1535 & 1551 & 1580 & 1595 & 1611 & 1635 & 1682 & 1736 & 1776 & 1896 \\
\hline & Lt.kid & 679 & 714 & 763 & 827 & 902 & 1060 & 1150 & 1202 & 1257 & 1360 & 1439 & 1493 & 1573 \\
\hline & Rt.kid & 1091 & 1142 & 1245 & 1338 & 1439 & 1617 & 1768 & 1830 & 1899 & 2036 & 2140 & 2210 & 2307 \\
\hline & Liver & 1294 & 1338 & 1395 & 1450 & 1504 & 1570 & 1632 & 1697 & 1759 & 1822 & 1893 & 1956 & 2032 \\
\hline & Max.Dose & 105 & 104.2 & 103.6 & 103.2 & 103.1 & 103.0 & 103 & 102.9 & 102.9 & 103.2 & 103.3 & 103.3 & 103.9 \\
\hline \multirow{6}{*}{$\begin{array}{l}4 \\
\text { Fields }\end{array}$} & PTV & 86.56 & 89.50 & 91.60 & 92.85 & 94.10 & 95.07 & 95.93 & 96.38 & 96.78 & 97.06 & 97.22 & 97.46 & 97.61 \\
\hline & S. Cord & 2710 & 2694 & 2685 & 2685 & 2689 & 2701 & 2707 & 2713 & 2727 & 2756 & 2791 & 2817 & 2894 \\
\hline & Lt.kid & 598 & 636 & 682 & 739 & 805 & 929 & 1010 & 1062 & 1118 & 1205 & 1278 & 1336 & 1409 \\
\hline & Rt.kid & 1030 & 1084 & 1205 & 1293 & 1383 & 1522 & 1664 & 1728 & 1800 & 1916 & 2011 & 2084 & 2184 \\
\hline & Liver & 1100 & 1141 & 1198 & 1250 & 1302 & 1369 & 1425 & 1489 & 1549 & 1610 & 1678 & 1741 & 1817 \\
\hline & Max Dose & 104.6 & 103.8 & 103.2 & 102.8 & 102.6 & 102.4 & 102.3 & 102.2 & 102.2 & 102.2 & 102.2 & 102.2 & 102.4 \\
\hline \multirow{6}{*}{$\begin{array}{l}5 \\
\text { Fields }\end{array}$} & PTV & 87.32 & 89.82 & 91.70 & 92.95 & 94.04 & 94.84 & 95.65 & 96.15 & 96.48 & 96.58 & 96.90 & 97.08 & 97.24 \\
\hline & S. Cord & 1852 & 1930 & 1993 & 2093 & 2123 & 2141 & 2173 & 2201 & 2238 & 2292 & 2351 & 2399 & 2501 \\
\hline & Lt.kid & 803 & 835 & 876 & 931 & 994 & 1120 & 1199 & 1246 & 1300 & 1386 & 1452 & 1501 & 1568 \\
\hline & Rt.kid & 1165 & 1211 & 1299 & 1383 & 1473 & 1622 & 1757 & 1812 & 1873 & 1988 & 2079 & 2144 & 2228 \\
\hline & Liver & 1183 & 1227 & 1283 & 1337 & 1395 & 1462 & 1526 & 1589 & 1650 & 1712 & 1784 & 1850 & 1925 \\
\hline & Max Dose & 104.7 & 103.9 & 103.4 & 103.2 & 103 & 102.8 & 102.7 & 102.6 & 102.5 & 102.6 & 102.7 & 102.8 & 102.9 \\
\hline
\end{tabular}

MLC difference margin start from $(0.0 \mathrm{~cm}$ to $1.2 \mathrm{~cm})$.

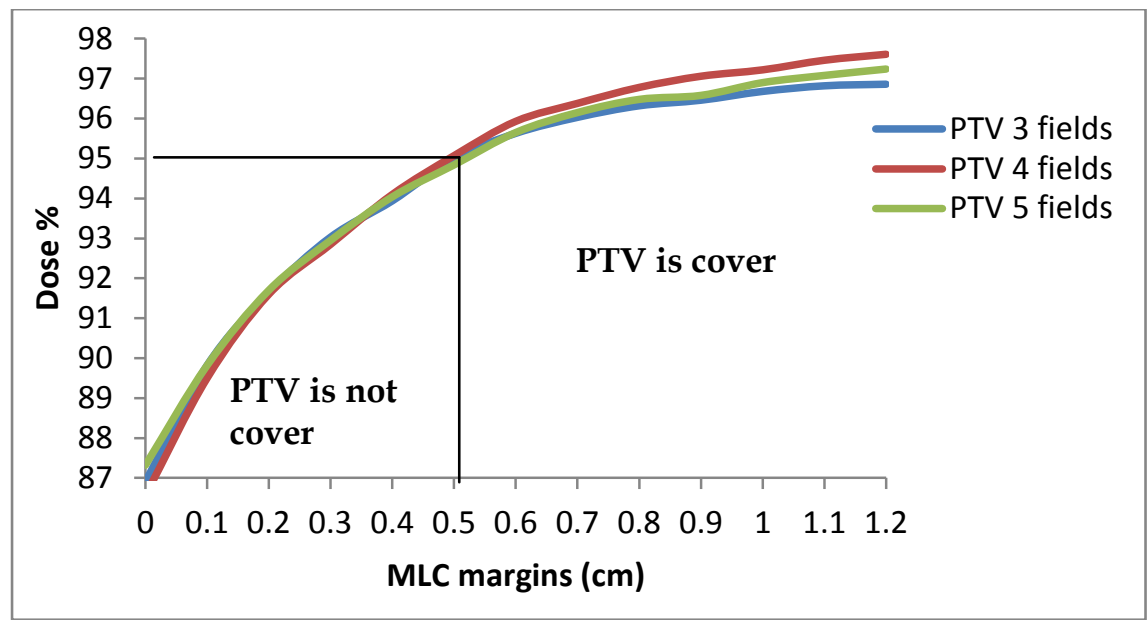

Fig. (11) PTV \& MLC margins.

The case No. 8

A woman (55 y.) with pancreas cancer, 


\begin{tabular}{|c|c|c|c|c|c|c|c|c|c|c|c|c|c|c|}
\hline Plan & MLC & 0.0 & 0.1 & 0.2 & 0.3 & 0.4 & 0.5 & 0.6 & 0.7 & 0.8 & 0.9 & 1.0 & 1.1 & 1.2 \\
\hline \multirow{6}{*}{$\begin{array}{l}3 \\
\text { Fields }\end{array}$} & PTV & 84.25 & 88.09 & 91.21 & 92.80 & 93.87 & 95.34 & 96.13 & 96.61 & 96.98 & 97.32 & 97.58 & 97.73 & 97.80 \\
\hline & S. Cord & 1881 & 1867 & 1910 & 1918 & 1923 & 1923 & 1981 & 1997 & 2008 & 2013 & 2059 & 2104 & 2784 \\
\hline & Lt.kid & 315 & 340 & 403 & 443 & 476 & 553 & 621 & 673 & 722 & 767 & 829 & 880 & 1001 \\
\hline & Rt.kid & 385 & 421 & 460 & 490 & 523 & 583 & 649 & 701 & 754 & 810 & 873 & 932 & 1013 \\
\hline & Liver & 1028 & 1086 & 1148 & 1200 & 1244 & 1311 & 1366 & 1417 & 1502 & 1556 & 1620 & 1680 & 1760 \\
\hline & Max Dose & 106 & 105 & 104 & 103.5 & 103.2 & 102.8 & 102.5 & 102.5 & 102.5 & 102.4 & 102.5 & 102.5 & 102.7 \\
\hline \multirow{6}{*}{$\begin{array}{l}4 \\
\text { Fields }\end{array}$} & PTV & 84.76 & 88.45 & 91.37 & 92.96 & 94.23 & 95.51 & 96.41 & 96.92 & 97.36 & 97.68 & 98.01 & 98.21 & 98.31 \\
\hline & S. Cord & 2601 & 2578 & 2602 & 2606 & 2599 & 2634 & 2638 & 2648 & 2654 & 2654 & 2693 & 2721 & 3252 \\
\hline & Lt.kid & 320 & 347 & 407 & 447 & 484 & 559 & 625 & 678 & 729 & 778 & 841 & 896 & 1006 \\
\hline & Rt.kid & 417 & 458 & 500 & 534 & 570 & 632 & 698 & 754 & 813 & 875 & 941 & 1006 & 1087 \\
\hline & Liver & 980 & 1037 & 1100 & 1144 & 1189 & 1253 & 1306 & 1356 & 1435 & 1494 & 1555 & 1614 & 1688 \\
\hline & Max Dose & 106.2 & 105.2 & 104.3 & 103.7 & \begin{tabular}{ll|}
103.3 \\
\end{tabular} & 102.8 & 102.5 & 102.4 & 102.3 & 102.4 & 102.5 & 102.5 & 102.4 \\
\hline \multirow{6}{*}{$\begin{array}{l}5 \\
\text { Fields }\end{array}$} & PTV & 85.44 & 88.67 & 91.49 & 92.96 & 94.13 & 95.39 & 96.21 & 96.69 & 97.02 & 97.36 & 97.66 & 97.88 & 97.98 \\
\hline & S. Cord & 2554 & 2556 & 2558 & 2574 & 2577 & 2612 & 2617 & 2628 & 2636 & 2639 & 2672 & 2687 & 3230 \\
\hline & Lt.kid & 560 & 583 & 634 & 668 & 700 & 763 & 821 & 867 & 908 & 951 & 1002 & 1051 & 1150 \\
\hline & Rt.kid & 504 & 540 & 578 & 606 & 641 & 697 & 758 & 808 & 855 & 913 & 972 & 1035 & 1110 \\
\hline & Liver & 1041 & 1102 & 1160 & 1208 & 1261 & 1322 & 1376 & 1426 & 1506 & 1562 & 1622 & 1686 & 1759 \\
\hline & Max.Dose & 106.2 & 105.2 & 104.3 & 103.8 & 103.4 & 103 & 102.8 & 102.6 & 102.8 & 102.8 & 102.7 & 102.7 & 102.7 \\
\hline
\end{tabular}

Table 13: a table illustrates all OAR value and PTV volume percentage cover for three different techniques with MLC difference margin start from $(0.0 \mathrm{~cm}$ to $1.2 \mathrm{~cm})$.

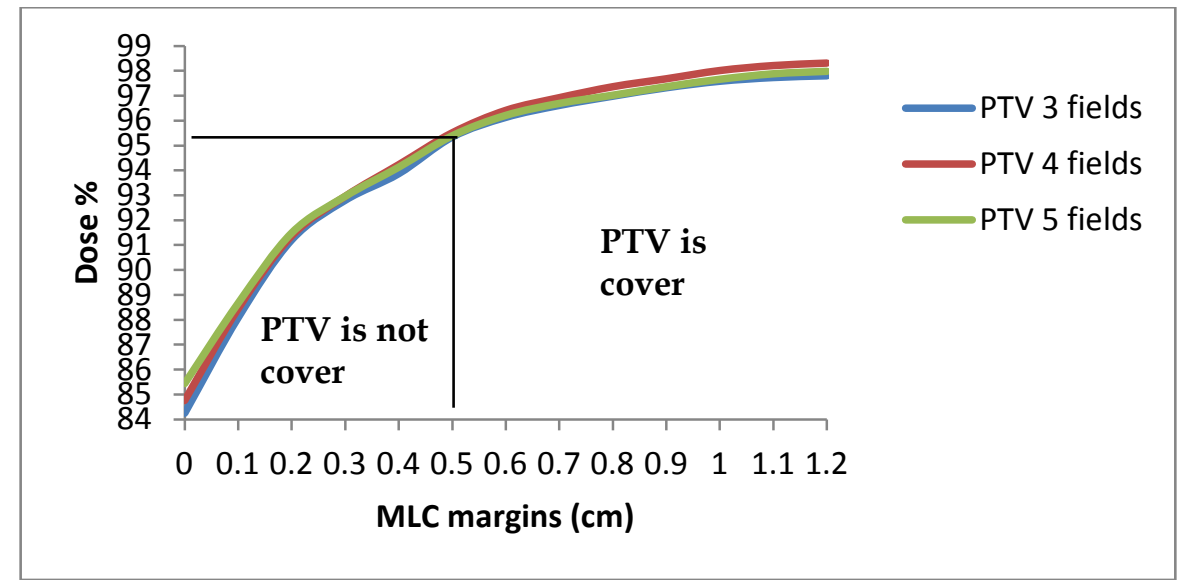

The case No. 9

Fig. (12) PTV \& MLC margins.

A man (43 y.) with pancreas cancer,

\begin{tabular}{|c|c|c|c|c|c|c|c|c|c|c|c|c|c|c|}
\hline Plan & MLC & 0.0 & 0.1 & 0.2 & 0.3 & 0.4 & 0.5 & 0.6 & 0.7 & 0.8 & 0.9 & 1.0 & 1.1 & 1.2 \\
\hline \multirow{6}{*}{$\begin{array}{l}3 \\
\text { Fields }\end{array}$} & PTV & 87.94 & 91.07 & 93.34 & 94.40 & 95.08 & 95.49 & 95.81 & 95.87 & 95.99 & 96.03 & 96.07 & 96.11 & 96.15 \\
\hline & S. Cord & 1122 & 1117 & 1116 & 1120 & 1122 & 1124 & 1131 & 1144 & 1147 & 1148 & 1154 & 1157 & 1162 \\
\hline & Lt.kid & 139 & 146 & 154 & 162 & 171 & 180 & 190 & 223 & 237 & 251 & 264 & 276 & 287 \\
\hline & Rt.kid & 235 & 246 & 262 & 278 & 299 & 318 & 336 & 357 & 377 & 403 & 424 & 441 & 462 \\
\hline & Liver & 1530 & 1600 & 1676 & 1747 & 1835 & 1905 & 1977 & 2033 & 2101 & 2174 & 2256 & 2333 & 2409 \\
\hline & Max Dose & 107.7 & 107 & 106.3 & 105.8 & 105.6 & 105.4 & 105.3 & 105.2 & 105.1 & 105.5 & 105.7 & 105.9 & 106 \\
\hline \multirow{6}{*}{$\begin{array}{l}4 \\
\text { Fields }\end{array}$} & PTV & 88.80 & 91.81 & 93.97 & 95.04 & 95.73 & 96.17 & 96.53 & 96.67 & 96.85 & 97.01 & 97.12 & 97.18 & 97.23 \\
\hline & S. Cord & 2981 & 2962 & 2942 & 2928 & 2918 & 2912 & 2910 & 2907 & 2904 & 2900 & 2805 & 2802 & 28 \\
\hline & Lt.kid & 166 & 177 & 188 & 200 & 212 & 226 & 239 & 255 & 280 & 305 & 365 & 400 & 455 \\
\hline & Rt.kid & 322 & 340 & 366 & 393 & 423 & 450 & 480 & 510 & 545 & 572 & 598 & 627 & 663 \\
\hline & Liver & 1502 & 1568 & 1639 & 1706 & 1794 & 1871 & 1940 & 2020 & 2103 & 2190 & 2275 & 2350 & 2434 \\
\hline & Max Dose & 106.1 & 105.4 & 104.8 & 104.4 & 104.2 & 104.0 & 103.8 & 103.5 & 103.5 & 103.6 & 103.7 & 103.9 & 104.2 \\
\hline \multirow{6}{*}{$\begin{array}{l}5 \\
\text { Fields }\end{array}$} & PTV & 89.17 & 91.63 & 92.94 & 93.85 & 94.44 & 94.80 & 95.06 & 95.14 & 95.23 & 95.30 & 95.40 & 95.45 & 95.48 \\
\hline & S. Cord & 990 & 1008 & 1023 & 1023 & 1112 & 1147 & 1320 & 1396 & 1623 & 1773 & 1788 & 1874 & 1927 \\
\hline & Lt.kid & 510 & 557 & 598 & 635 & 687 & 727 & 778 & 826 & 869 & 930 & 969 & 1027 & 1074 \\
\hline & Rt.kid & 170 & 183 & 195 & 208 & 226 & 240 & 269 & 287 & 301 & 321 & 337 & 358 & 377 \\
\hline & Liver & 1199 & 1263 & 1322 & 1385 & 1442 & 1501 & 1574 & 1640 & 1704 & 1777 & 1839 & 1903 & 1966 \\
\hline & Max.Dose & 107.4 & 106.7 & 106.2 & 106 & 105.8 & 105.6 & 105.8 & 106.1 & 106.1 & 106.1 & 106.2 & 106.4 & 106.5 \\
\hline
\end{tabular}

Table 14: a table illustrates all OAR value and PTV volume percentage cover for three different techniques with MLC difference margin start from $(0.0 \mathrm{~cm}$ to $1.2 \mathrm{~cm})$. 


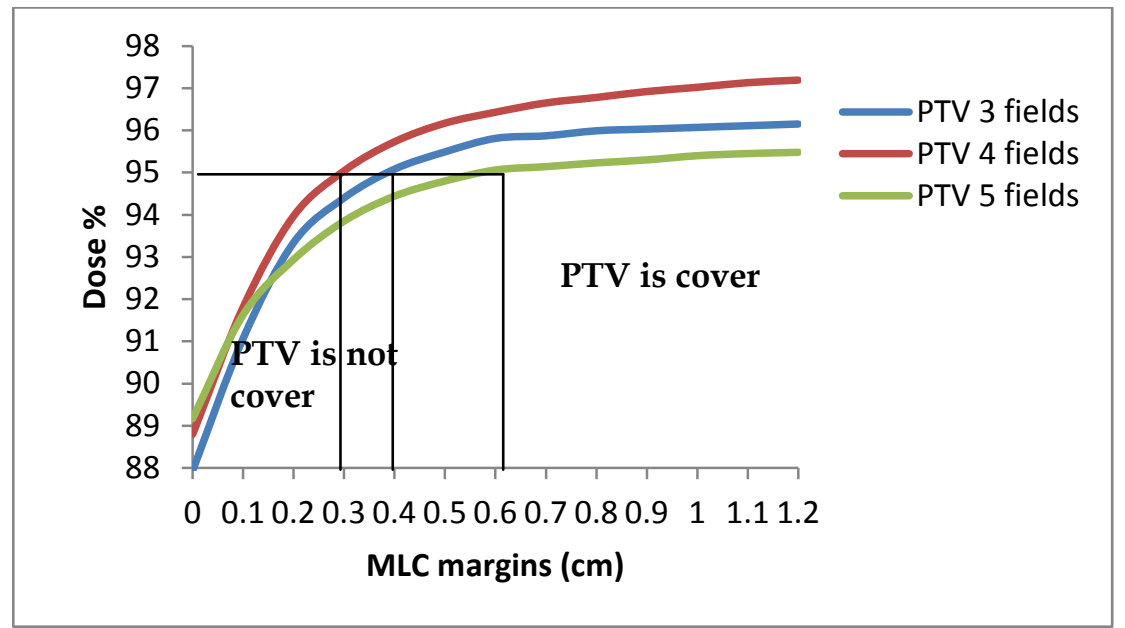

Fig. (13) PTV \& MLC margins.

The case No. 10

A man (62 y.) with pancreas cancer,

\begin{tabular}{|c|c|c|c|c|c|c|c|c|c|c|c|c|c|c|}
\hline Plan & MLC & 0.0 & 0.1 & 0.2 & 0.3 & 0.4 & 0.5 & 0.6 & 0.7 & 0.8 & 0.9 & 1.0 & 1.1 & 1.2 \\
\hline \multirow{6}{*}{$\begin{array}{l}3 \\
\text { Fields }\end{array}$} & PTV & 87.30 & 90.20 & 92.50 & 94.00 & 94.84 & 95.30 & 95.80 & 95.99 & 96.20 & 96.26 & 96.30 & 96.37 & 96.45 \\
\hline & S. Cord & 2442 & 2483 & 3475 & 3508 & 3531 & 3545 & 3579 & 4693 & 4720 & 4746 & 4769 & 4794 & 4910 \\
\hline & Lt.kid & 1454 & 1517 & 1886 & 1968 & 2020 & 20.86 & 2150 & 2429 & 2496 & 2555 & 2630 & 2710 & 3150 \\
\hline & Rt.kid & 699 & 747 & 967 & 1020 & 1065 & 1113 & 1165 & 1325 & 1378 & 1432 & 1490 & 1560 & 1860 \\
\hline & Liver & 1308 & 1350 & 1400 & 1446 & 1488 & 1538 & 1586 & 1652 & 1710 & 1760 & 1810 & 1860 & 1910 \\
\hline & Max Dose & 107.1 & 106.2 & 105.6 & 105.2 & 105.1 & 105 & 105 & 105 & 105 & 105.2 & 105.3 & 105.3 & 105.7 \\
\hline \multirow{6}{*}{$\begin{array}{l}4 \\
\text { Fields }\end{array}$} & PTV & 87.6 & 90.4 & 92.5 & 94.3 & 94.9 & 95.5 & 96.0 & 96.25 & 96.6 & 96.85 & 96.9 & 97.05 & 97.2 \\
\hline & S. Cord & 3012 & 3132 & 3962 & 3986 & 4050 & 4102 & 4153 & 4750 & 4785 & 4814 & 4856 & 4877 & 4955 \\
\hline & Lt.kid & 1534 & 1617 & 2006 & 2068 & 2100 & 2176 & 2216 & 2329 & 2456 & 2585 & 2630 & 2695 & 3166 \\
\hline & Rt.kid & 720 & 787 & 997 & 1080 & 1106 & 1145 & 1197 & 1345 & 1398 & 1449 & 1500 & 1566 & 1732 \\
\hline & Liver & 1328 & 1355 & 1430 & 1476 & 1495 & 1555 & 1596 & 1676 & 1730 & 1785 & 1842 & 1884 & 1935 \\
\hline & Max Dose & 107 & 106.2 & 105.4 & 105 & 104.9 & 104.8 & 104.7 & 104.5 & 104.5 & 104.5 & 104.5 & 104.6 & 104.6 \\
\hline \multirow{6}{*}{$\begin{array}{l}5 \\
\text { Fields }\end{array}$} & PTV & 87.8 & 90.3 & 92.75 & 94.2 & 95 & 95.6 & 96.1 & 96.4 & 96.8 & 96.9 & 97.1 & 97.4 & 97.5 \\
\hline & S. Cord & 2946 & 2972 & 3662 & 3686 & 3700 & 3727 & 3751 & 4650 & 4688 & 4716 & 4754 & 4774 & 4855 \\
\hline & Lt.kid & 1703 & 1757 & 2076 & 2150 & 2190 & 2250 & 2310 & 2547 & 2605 & 2658 & 2725 & 2791 & 3178 \\
\hline & Rt.kid & 860 & 900 & 1060 & 1107 & 1152 & 1192 & 1240 & 1373 & 1419 & 1467 & 1520 & 1580 & 1810 \\
\hline & Liver & 1282 & 1324 & 1371 & 1420 & 1470 & 1520 & 1560 & 1627 & 1679 & 1731 & 1784 & 1837 & 1885 \\
\hline & Max.Dose & 107.2 & 106.4 & 105.7 & 105.3 & 105 & 104.8 & 104.6 & 104.7 & 104.7 & 104.7 & 104.7 & 104.7 & 104.6 \\
\hline
\end{tabular}

Table 15: a table illustrates all OAR value and PTV volume percentage cover for three different techniques with MLC difference margin start from $(0.0 \mathrm{~cm}$ to $1.2 \mathrm{~cm})$.

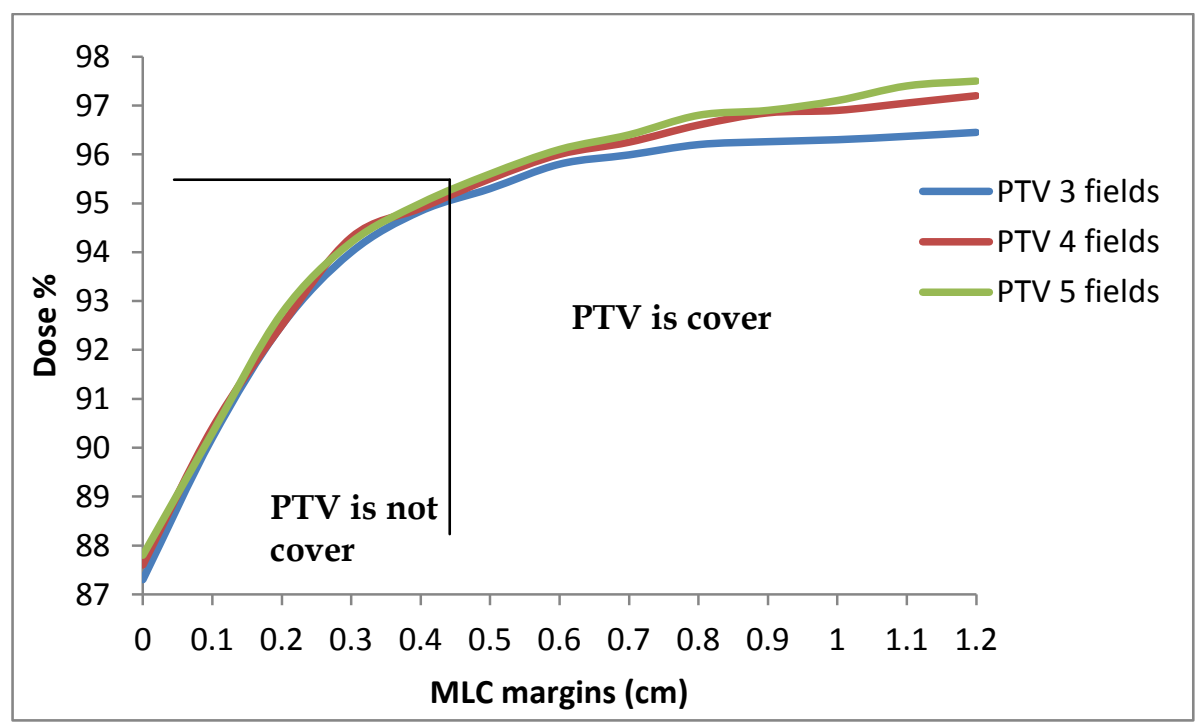

Fig. (14) PTV \& MLC margins. 


\begin{tabular}{|c|c|c|c|c|c|c|c|c|c|c|c|c|c|}
\hline MLC & 0.0 & 0.1 & 0.2 & 0.3 & 0.4 & 0.5 & 0.6 & 0.7 & 0.8 & 0.9 & 1.0 & 1.1 & 1.2 \\
\hline PTV & 86.98 & 89.99 & 92.23 & 93.70 & 94.70 & 95.51 & 96.10 & 96.49 & 96.79 & 97.02 & 97.17 & 97.33 & 97.39 \\
\hline
\end{tabular}

The following table contains the averages for each margin for all of three techniques compound for PTV.

Table 16: a table illustrates the averages of PTV volume percentage cover with MLC difference margin start from $(0.0 \mathrm{~cm}$ to $1.2 \mathrm{~cm})$.

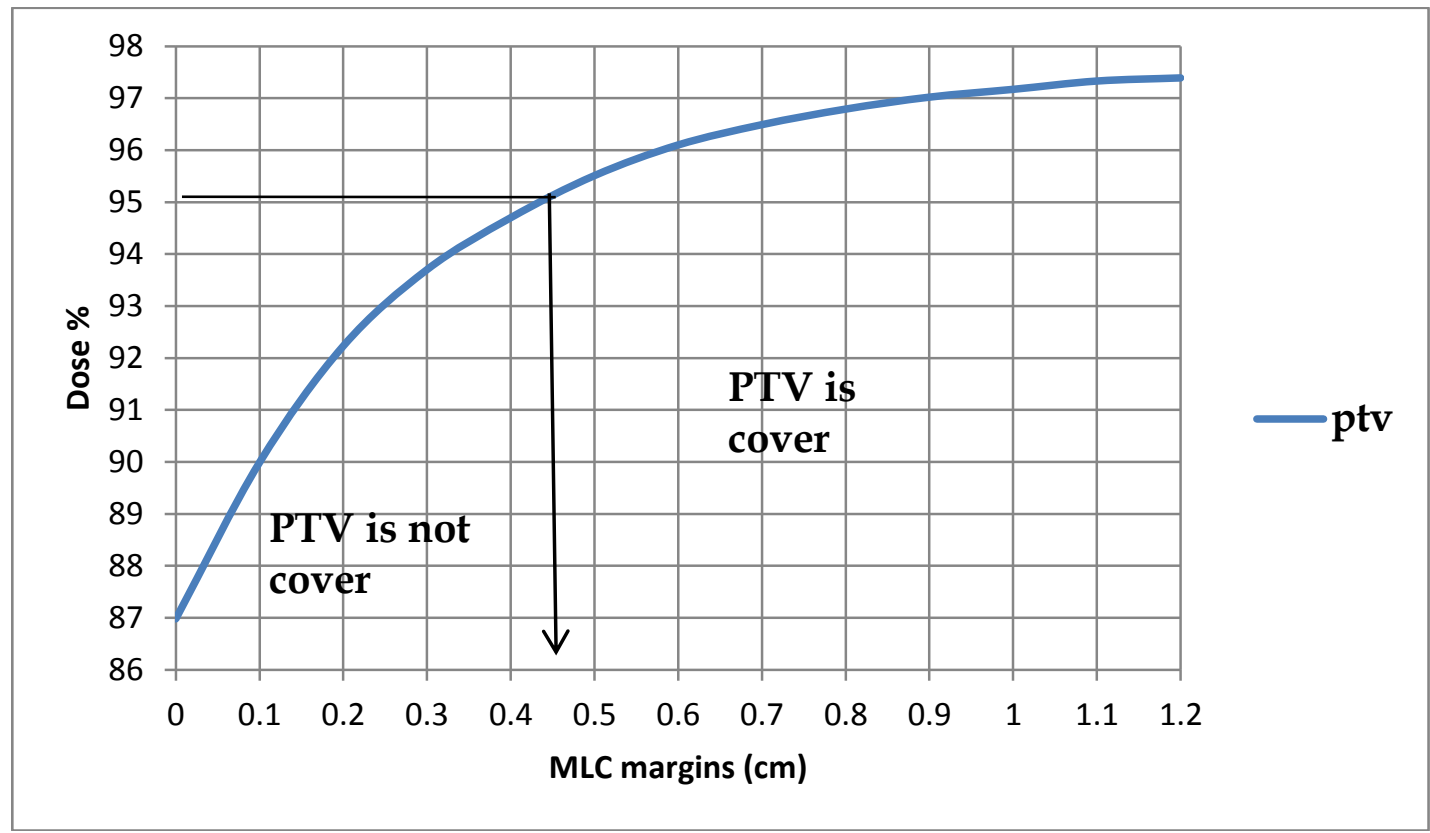

Fig. (15) PTV \& MLC margins.

\section{Conclusions}

As field size increase as the dose of target point's increase and the dose distribution become better for tumor but OAR become worst. This fact is true for regular field size which shaped by collimator jaws only (square and rectangular) and true for irregular field size which shaped by custom block or MLCs (circular and irregular) too.

The plans with MLCs margin from $0.0 \mathrm{~cm}$ to $0.4 \mathrm{~cm}$ are not accepted because the $95 \%$ of dose doesn't cover $95 \%$ of tumor volume and this may lead to the occurrence of recurrence. The plans with MLCs margin 0.5 $\mathrm{cm}, 0.6 \mathrm{~cm}$ and $0.7 \mathrm{~cm}$ are the best plans because the $95 \%$ of dose cover more than $95 \%$ of tumor volume and OAR still under tolerance. Although, the $95 \%$ of dose cover more than $95 \%$ of tumor volume, the plans with MLCs margin more than $0.7 \mathrm{~cm}$ are not accepted because the OAR received unjustified and unnecessary dose and more than tolerance for sometimes.

\section{References}

[1]. Khan, Faiz M.(2010) Physics of Radiation Therapy, The, 4th Edition: Lippincott Williams \& Wilkins.

[2]. Murat Beyzadeoglu , Gokhan Ozyigit, Cuneyt Ebruli (2010) Basic Radiation Oncology: Springer-Verlag Berlin Heidelberg 2010.

[3]. Podgorsak EB (2005) Radiation oncology physics: a handbook for teachers and students. International Atomic Energy Agency, Vienna.

[4]. Podgoršak E (2007) Radiation physics for medical physicists, 1st edn. Springer, Berlin.

[5]. Cormack DV, Johns HE. Spectral distribution of scattered radiation from a kilocurie cobalt 60 unit. Br J Radiol. 1958.

[6]. Pam Cherry MSc TDCR, Angela M. Duxbury MSc FCR TDCR (2009) Practical radiotherapy: physics and equipment -2 nd Ed. This edition first published 2009. 
[7]. Powers WE, Kinzie JJ, Demidecki AJ, et al. A new system of field shaping for external-beam radiation therapy. Radiology. 1973.

[8]. Earl JD, Bagshaw MA. A rapid method for preparation of complex field shapes. Radiology. 1967.

[9]. Edland RW, Hansen H. Irregular field-shaping for 60Co teletherapy. Radiology. 1969. 